\title{
Koopmans-Compliant Spectral Functionals for Extended Systems
}

\author{
Ngoc Linh Nguyen, ${ }^{1, *}$ Nicola Colonna, ${ }^{1}$ Andrea Ferretti, ${ }^{2}$ and Nicola Marzari ${ }^{1}$ \\ ${ }^{1}$ Theory and Simulations of Materials (THEOS), and National Centre for Computational Design \\ and Discovery of Novel Materials (MARVEL), École Polytechnique Fédérale de Lausanne, \\ 1015 Lausanne, Switzerland \\ ${ }^{2}$ Centro S3, CNR-Istituto Nanoscienze, 41125 Modena, Italy
}

(Received 22 August 2017; revised manuscript received 31 January 2018; published 23 May 2018)

\begin{abstract}
Koopmans-compliant functionals have been shown to provide accurate spectral properties for molecular systems; this accuracy is driven by the generalized linearization condition imposed on each charged excitation, i.e., on changing the occupation of any orbital in the system, while accounting for screening and relaxation from all other electrons. In this work, we discuss the theoretical formulation and the practical implementation of this formalism to the case of extended systems, where a third condition, the localization of Koopmans's orbitals, proves crucial to reach seamlessly the thermodynamic limit. We illustrate the formalism by first studying one-dimensional molecular systems of increasing length. Then, we consider the band gaps of 30 paradigmatic solid-state test cases, for which accurate experimental and computational results are available. The results are found to be comparable with the state of the art in many-body perturbation theory, notably using just a functional formulation for spectral properties and the generalizedgradient approximation for the exchange and correlation functional.
\end{abstract}

DOI: 10.1103/PhysRevX.8.021051

\section{INTRODUCTION}

Accurate first-principles predictions of spectral properties - such as band gaps or photoemission spectraattract considerable attention because of their critical role in the design and characterization of optical and electronic devices, e.g., for solar energy harvesting and conversion [1]. To date, the most common approaches to compute these quantities in extended systems are based on many-body perturbation theory (MBPT) using Green's-function-based approaches [2] (such as the GW approximation [3]) or wavefunction-based methods like coupled cluster [4] or quantum Monte Carlo [5], with GW being considered for the case of solids a good compromise between accuracy and computational costs. Nevertheless, these high-level methods are still significantly limited in system size and complexity, due to their computational costs, and even in accuracy, at least for the most common techniques. For these reasons, simpler methods based on Kohn-Sham density-functional theory [6,7] (KS-DFT), possibly including some fraction of nonlocal exchange [8], are still frequently employed to evaluate

\footnotetext{
*linh.nguyen@epfl.ch
}

Published by the American Physical Society under the terms of the Creative Commons Attribution 4.0 International license. Further distribution of this work must maintain attribution to the author(s) and the published article's title, journal citation, and DOI.

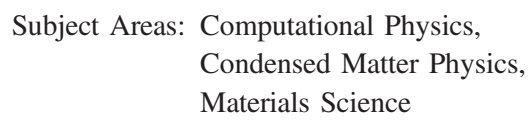

approximately the spectral properties of nanostructures, interfaces, or solids.

In fact, exact KS-DFT would correctly predict the energy of the highest occupied eigenstate, since this determines the long-range decay of the charge density into vacuum, which needs to be described correctly in exact KS-DFT $[9,10]$ (see also Ref. [11] and references therein for an indepth discussion); all other eigenstates and spectral properties remain outside the domain of KS-DFT. In particular, the lowest unoccupied eigenstate is not meant to be correctly positioned, even in the exact theory, thus always leading to incorrect fundamental gaps. Notably, when the lowest unoccupied eigenstate becomes infinitesimally occupied, it needs to jump to the correct highest occupied eigenvalue, explaining why the exact KS potential has a derivative discontinuity as a function of orbital occupations [12-14]. Approximate KS-DFT (especially in the local or generalized-gradient approximations) compounds these issues, and both frontier orbital energies become incorrectly positioned-typically raising the highest occupied eigenstate, lowering the lowest unoccupied one, and further decreasing the fundamental gap. These considerations extend also to solids, and, e.g., the band gap energy $E_{g}$ is often greatly underestimated [14].

Such failures have been connected to the deviation from piecewise linearity (PWL) of the total energy functional as a function of particle number, and the associated lack of derivative discontinuities at integer occupation (this PWL 
condition, thanks to Janak's theorem [15], is equivalent to stating that the energy of the highest occupied orbital needs to be independent from the occupation of the orbital itself). Deviations from PWL have also been suggested [16-20] to be driven by electronic self-interaction errors (SIEs) [21], and in recently developed functionals, such as rangeseparated [22,23] or dielectric-dependent hybrid functionals [24,25], PWL has been recognized as a critical feature to address. The criterion of piecewise linearity was chosen in particular as a key feature by some of us when introducing the class of Koopmans-compliant (KC) functionals [26-30] that enforce a generalized criterion of PWL; in these approaches, every orbital energy is independent from the occupation of the orbital itself (we note in passing that these functionals generalize to all orbitals the linearization criterion of the DFT + Hubbard $U$ approach [16,17], which linearizes the energy with respect to the occupation of the Hubbard manifold). The accuracy of $\mathrm{KC}$ functionals in reproducing spectral properties is quite remarkable [29-32] and, in our view, it highlights the role of these functionals as approximations to the exact spectral functional, i.e., the functional able to reproduce spectral properties in addition to total energies [33]. Last, we also mention that the potential energy surface of $\mathrm{KC}$ functionals preserves exactly or slightly improves [32] the base functional upon which they are constructed (typically, the PBE [34] approximation is used). We briefly summarize and expand here the concepts alluded to above; a detailed description of the KC spectral functionals, first introduced in Refs. [26,27], can be found in Refs. [30,35]. The three core concepts that underpin their formulation are those of linearization, screening, and localization.

The first concept of linearization refers to the generalization of the PWL condition of Perdew et al. [36]. Before doing that, we note that PWL in the total energy with respect to the total number of particles is equivalent to PWL in the total energy with respect to the occupation of the highest-energy state, and it is also equivalent to having a constant orbital energy (i.e., an expectation value on the KS Hamiltonian) for the highest-energy state as a function of its occupation-all of this thanks to Janak's theorem [15]. Then, the generalized PWL condition that $\mathrm{KC}$ functionals introduce is one where there is a constant orbital energy for any orbital in the system, rather than the highest-energy one only, irrespectively of the fractional occupation of that orbital. This condition can also be seen as a definition of being self-interaction free: If the orbital energy does not depend on the occupation of the orbital itself, that orbital is free from self-interactions [27]. So, if one takes the representation where the single-particle Hamiltonian is diagonal (from now on referred to as "canonical" representation), this generalized condition of PWL when extracting an electron from an orbital is naturally akin to that of (i) removing - heuristically - the self-interaction contributions of that orbital from the functional and (ii) allowing us to interpret single-particle eigenvalues as charged excitation energies. This second point can be understood by noting that the generalized PWL condition in combination as above with Janak's theorem enforces the orbital energy (i.e., the expectation value of the Hamiltonian on a given orbital) to be equal to the energy difference between the system with $N$ electrons and that with $N-1$ electrons, where one electron has been removed from that orbital; this is the definition of a charged excitation.

The discussion above is general, but it does not take into account explicitly relaxations, i.e., the response of all the electrons as the occupation of one orbital is changed. This is where the second core concept of screening arises. In the $\mathrm{KC}$ formalism, this is accounted for by introducing a screening coefficient in front of a "bare" KC correction designed to enforce the generalized PWL condition described above in a frozen-orbitals picture, i.e., in a framework where the relaxation of the orbitals is completely neglected [for the definition, see Eq. (1) in the next section]. In the first applications to atomic and molecular systems [27,29-31], screening was approximately accounted for by using one single screening coefficient to the Koopmans's corrections for all filled orbitals, and one for all empty orbitals. These two coefficients were determined, respectively, by the condition that the energy of the highest occupied molecular orbital (HOMO) of the neutral system needs to be equal to the energy of the lowest unoccupied molecular orbital (LUMO) of the cation (providing the screening coefficient for all filled orbitals) and that the LUMO eigenvalue of the neutral system needs to be equal to the HOMO eigenvalue of the anion (providing the screening coefficient for the empty orbitals). While this approximation can be satisfactory and even accurate for small, simple molecules, an orbital-dependent formalism calls for orbital-dependent screening; in addition, it is essential to transition these concepts correctly in the thermodynamic limit of a solid, as will be discussed in the paper. We note in passing that these screening coefficients can be calculated using finite differences (as done here and detailed below) or, more elegantly, using linear-response theory (detailed in Ref. [37]).

The third concept of localization becomes truly determinant in the thermodynamic limit, i.e., for extended systems: The condition of Koopmans's compliance relies in an essential way on localization when considering larger and larger systems, where the variational Koopmans's orbitals converge rapidly to their thermodynamic limit, which is localized (for the sake of illustration, they closely resemble maximally localized Wannier functions [38]). This point will be discussed in detail in the second part of the paper.

The fact that Koopmans's compliance can lead to orbital energies that can be compared to the quasiparticle excitation energies of photoemission experiments, and to canonical orbitals that resemble Dyson orbitals, has been discussed extensively for the case of molecular systems [33]. In previous work [29,31,32], we presented 
the performance of $\mathrm{KC}$ functionals in predicting frontier energies, ultraviolet photoemission spectra, and orbital tomography momentum maps for different classes of molecules, while also arguing that these functionals provide accurate quasiparticle approximations to the exact spectral potential $[33,39]$. In fact, we typically find very good agreement with experiments, comparable or sometimes even better than state-of-the-art MBPT methods, while preserving moderate computational costs and the quality of the potential energy surface of the underlying base functional [30] (or even improving on it when using the KIPZ implementation of KC functionals [32]). In this work, we discuss how the framework of $\mathrm{KC}$ functionals extends to the case of solids. We focus first on the conceptual issues, and then on the calculation of energy gaps and IP energies (for surfaces). In Sec. II, we describe the main theoretical challenges and the approach adopted in this work. In Sec. II C, we study finite alkane chains of increasing length and discuss the thermodynamic limit in these one-dimensional systems. Then, in Sec. III A, we assess the method against the calculation of band gaps in 3D semiconductors and insulators, as well as selected surfaces. The accuracy in predicting $E_{g}$ and IP energies is compared to experiments, standard KS-DFT calculations, many-body perturbation theory, and coupled-cluster $[\operatorname{CCSD}(\mathrm{T})]$ wave function methods.

\section{THEORY AND METHODS}

\section{A. Linearization in Koopmans-compliant functionals}

As mentioned, KC functionals [26-30,40,41] explicitly enforce generalized PWL conditions to an entire electronic manifold, introducing functionals that aim at having a linear dependence of the energy as a function of the occupation of any orbital in the system. Formally, these functionals are constructed starting from the exact or an approximate DFT energy functional $E^{\mathrm{DFT}}$ (the "base" functional) and replacing, orbital by orbital, the contribution to the total DFT energy that is not linear in the fractional orbital occupation with one that is linear [this entails subtracting out, for each orbital, the Slater integral, i.e., the opposite of the first term in the right-hand side of Eq. (2), and adding in lieu of that a linear $f_{i} \eta_{i}$ Koopmans's term]:

$$
\begin{aligned}
& E^{\mathrm{KC}}=E^{\mathrm{DFT}}+\sum_{i} \alpha_{i} \Pi_{i}^{\mathrm{KC}}, \\
& \Pi_{i}^{\mathrm{KC}}=-\int_{0}^{f_{i}}\left\langle\varphi_{i}\left|\hat{H}^{\mathrm{DFT}}(s)\right| \varphi_{i}\right\rangle d s+f_{i} \eta_{i} .
\end{aligned}
$$

Here, $\hat{H}^{\mathrm{DFT}}(s)$ is the KS Hamiltonian of the base functional (exact or approximated) calculated with a fractional occupation $s$ in orbital $\varphi_{i}$, and $\alpha_{i}$ are screening coefficients introduced to ensure that the linearity is preserved when taking into account the response and relaxation of all other orbitals $\left\{\varphi_{j \neq i}\right\}$. The slope $\eta_{i}$ in the linear Koopmans's term can be chosen in a number of ways, leading to different KC flavors. In this work, we focus on the KI and KIPZ implementations, described in detail in Ref. [30]. In KI, the slope $\eta_{i}$ is chosen as the total energy difference of two adjacent electronic configurations with integer occupations:

$$
\begin{aligned}
\eta_{i}^{\mathrm{KI}} & =E^{\mathrm{DFT}}\left[f_{i}=1\right]-E^{\mathrm{DFT}}\left[f_{i}=0\right] \\
& =\int_{0}^{1}\left\langle\varphi_{i}\left|\hat{H}^{\mathrm{DFT}}(s)\right| \varphi_{i}\right\rangle d s ;
\end{aligned}
$$

the explicit expression for the unscreened KI Koopmans's correction becomes thus

$\Pi_{i}^{\mathrm{KI}}=-\int_{0}^{f_{i}}\left\langle\varphi_{i}\left|\hat{H}^{\mathrm{DFT}}(s)\right| \varphi_{i}\right\rangle d s+f_{i} \int_{0}^{1}\left\langle\varphi_{i}\left|\hat{H}^{\mathrm{DFT}}(s)\right| \varphi_{i}\right\rangle d s$,

where it can be seen that at integer occupations $\Pi_{i}^{\mathrm{KI}}=0$, and the KI functional becomes identical to its base functional, independently from the screening coefficients. The KI functional thus preserves exactly the potential energy surface of the base functional it started from, including that of the exact DFT functional (if one had it); its values at fractional occupations are instead different (except when the highest occupied state is concerned), and so are the derivatives calculated at integer occupations-hence, the effect on spectral properties.

In KIPZ, the slope $\eta_{i}$ is also chosen as the total energy difference of two adjacent electronic configurations with integer occupations, but this time using the Perdew-Zunger (PZ) self-interaction corrected (SIC) functional [21] applied onto the approximate DFT base functional. We have

$$
\begin{aligned}
\eta_{i}^{\mathrm{KIPZ}} & =E^{\mathrm{PZ}}\left[f_{i}=1\right]-E^{\mathrm{PZ}}\left[f_{i}=0\right] \\
& =\int_{0}^{1}\left\langle\varphi_{i}\left|\hat{H}_{i}^{\mathrm{PZ}}(s)\right| \varphi_{i}\right\rangle d s,
\end{aligned}
$$

providing the explicit expression for the unscreened $\Pi_{i}^{\mathrm{KIPZ}}$ correction [42],

$$
\begin{aligned}
\Pi_{i}^{\mathrm{KIPZ}}= & -\int_{0}^{f_{i}}\left\langle\varphi_{i}\left|\hat{H}^{\mathrm{DFT}}(s)\right| \varphi_{i}\right\rangle d s \\
& +f_{i} \int_{0}^{1}\left\langle\varphi_{i}\left|\hat{H}_{i}^{\mathrm{PZ}}(s)\right| \varphi_{i}\right\rangle d s,
\end{aligned}
$$

where $\hat{H}_{i}^{\mathrm{PZ}}(s)=\hat{H}^{\mathrm{DFT}}(s)-\hat{v}_{\mathrm{Hxc}}^{\mathrm{DFT}}\left[s\left|\varphi_{i}(\mathbf{r})\right|^{2}\right]$, with $-\hat{v}_{\mathrm{Hxc}}^{\mathrm{DFT}}\left[s \mid \varphi_{i}\right.$ $\left.(\mathbf{r})\right|^{2}$ the PZ self-interaction correction for the $i$ th orbital, which subtracts out the sum of the Hartree and exchangecorrelation potentials for that orbital, which has occupation $s$ and orbital density $s\left|\varphi_{i}(\mathbf{r})\right|^{2}$. Note that, in the unscreened case $\left[\alpha_{i}=1\right.$ in Eq. (1)], the KIPZ functional can be thought 
of as the KI correction applied to the PZ-SIC functional [this can be verified by replacing the base DFT functional and Hamiltonian in Eqs. (1)-(4) with their PZ-SIC counterparts]. However, in the general case of $\alpha_{i} \neq 1$, the KIPZ functional form Eq. (6) implies also scaling each PZ selfinteraction correction with its own screening coefficient. At integer occupations, the KIPZ functional thus results in a scaled PZ-SIC functional; this is a desirable property, since the bare PZ self-interaction correction tends to overcorrect the base functional $[43,44]$, and introducing a scaling parameter often improves the energetics and thermochemistry [45-49]. The KIPZ screening coefficients thus play a dual role; they ensure linearity when taking into account orbital relaxations and act as physically motivated scaling parameters for the $\mathrm{PZ}$ corrections [50].

In summary, these two approaches lead to two functionals that depend only on orbital densities (hence, these are orbital-density-dependent functionals), with the following final expressions for the unscreened KI and KIPZ corrections [30]:

$$
\begin{aligned}
\Pi_{i}^{\mathrm{KI}} & =E_{\mathrm{Hxc}}\left[\rho-\rho_{i}\right]-E_{\mathrm{Hxc}}[\rho]+f_{i}\left(E_{\mathrm{Hxc}}\left[\rho-\rho_{i}+n_{i}\right]\right. \\
& \left.-E_{\mathrm{Hxc}}\left[\rho-\rho_{i}\right]\right), \\
\Pi_{i}^{\mathrm{KIPZ}} & =\Pi_{i}^{\mathrm{KI}}-f_{i} E_{\mathrm{Hxc}}\left[n_{i}\right],
\end{aligned}
$$

having defined $\rho_{i}(\mathbf{r})=f_{i}\left|\varphi_{i}(\mathbf{r})\right|^{2}$ and $n_{i}(\mathbf{r})=\left|\varphi_{i}(\mathbf{r})\right|^{2}$, and with $E_{\mathrm{Hxc}}$ denoting the Hartree and exchange-correlation energy corresponding to the underlying base functional. For all calculations presented in this work, the base functional is PBE [34]. As mentioned, the orbital-dependent screening coefficients $\alpha_{i}$ account for the electronic screening and orbital relaxation; if these were all set to be equal to 1, the KC functionals in Eq. (1) would fulfill the Koopmans condition at frozen orbitals [28], rather than at relaxed orbitals.

The generalized linearity condition enforced by the KC functionals is more stringent than the piecewise linearity condition satisfied by the exact KS-DFT ground-state energy. The latter holds when the occupation of the highest occupied state (or, equivalently, the total number of particles) is varied, while the former applies to any orbital in the system. This, in turn, leads to a more general orbitaldensity-dependent framework where, at variance with DFT functionals but similarly to the other orbital-densitydependent methods, such as the PZ self-interaction correction itself [21,51], the functionals are not invariant under unitary transformations within the manifold of filled orbitals. In these cases, the variational orbitals $\left\{\left|\varphi_{i}\right\rangle\right\}$ that minimize the functional are different from the eigenstates or canonical orbitals $\left\{\left|\phi_{m}\right\rangle\right\}$ that diagonalize the orbitaldensity-dependent Hamiltonian, as discussed, e.g., in Refs. [30,31,51-55]. The algorithm that we advocate to minimize these functionals consists of two nested steps [35], following the ensemble-DFT approach [56]: First, (i) a minimization is performed with respect to all unitary transformations of the orbitals (the so-called "inner loop"; this minimization enforces the Pederson condition $\left\langle\varphi_{i} \mid \hat{v}_{j}^{\mathrm{KC}} \varphi_{j}\right\rangle=\left\langle\varphi_{i} \hat{v}_{i}^{\mathrm{KC}} \mid \varphi_{j}\right\rangle \quad$ with $\quad \hat{v}_{i}^{\mathrm{KC}}=\left[\delta /\left(\delta \rho_{i}\right)\right] \sum_{j} \Pi_{j}^{\mathrm{KC}}$, first discussed in Refs. [53,57]). Then, (ii) a variational optimization of the orbitals (outer loop) is performed using a standard conjugated-gradient strategy.

Koopmans's compliance from Eqs. (1)-(8) can be imposed on both valence and conduction orbitals. Currently, the only requirement is that the system under consideration needs to have a finite gap, which ensures that the occupation of any variational orbital is either 0 or 1 , and the definition of the corresponding orbital densities $\rho_{i}(\mathbf{r})$ is unambiguous (the occupation number of a variational orbital that is a mix of filled canonical orbitals, or of empty canonical orbitals, is an eigenvalue and keeps the occupation matrix diagonal, but this is not so, if filled and empty canonical orbitals are mixed together). So, any matrix element of the KC Hamiltonian between filled and empty orbitals is projected out [28], and the minimization can be performed separately for each manifold (still keeping orbitals orthogonal).

When working on the occupied manifold, the KIPZ functional leads to a set of well-localized variational orbitals. This is also true for the KI functional, since it is defined as the limit of the KIPZ functional with zero PZ correction [30] (otherwise, thanks to its unitary invariance at integer occupation, KI does not have a preferred representation). On the other hand, the empty orbitals are often delocalized [58]. Now, calculating the Koopmans corrections on a localized set of orbitals $\left\{\left|\varphi_{i}\right\rangle\right\}$ is a key requirement to deal with extended systems, since one wants the $\Pi_{i}^{\mathrm{KC}}$ corrections to remain finite (rather than tend to zero) and to converge rapidly to their thermodynamic limit. A workaround for the delocalization of empty orbitals in the current functionals is to compute a non-self-consistent but screened Koopmans correction using maximally localized Wannier orbitals as the localized representation for the lower part of the manifold of empty orbitals [59]. Even though this choice is arbitrary, it can provide a practical and effective scheme, as clearly supported by the results of the present work. Moreover, this choice does not affect the occupied orbitals and, thus, preserves the potential energy surface of the KI or KIPZ functional. We note that a similar treatment has also been introduced recently by Ma and Wang in Ref. [61]. In this work, the authors correct the band gap of semiconductors and insulators by enforcing a straight-line condition of the total energy upon removal (addition) of a fractional electron on Wannier functions constructed within the occupied (empty) manifolds; i.e., they perform a KI correction on Wannier functions.

\section{B. Screening in Koopmans-compliant functionals}

The bare Koopmans correction enforces the linearity of the energy under the assumption that single-particle orbitals 
are frozen upon changing one of the occupation numbers. In reality, one wants to include the response of the system to the ionization process (change of occupation) described by the Koopmans correction, and we account for this response (i.e., the screening) by introducing the orbital-dependent coefficients $\alpha_{i}$ in the definition of the $\mathrm{KC}$ functional in Eq. (1). As mentioned earlier, for small molecules [31,32], it is typically sufficient to compute two values of $\alpha$, one to be used for all the occupied orbitals, and the other to be used for all empty orbitals; these two values can be chosen by enforcing that the HOMO eigenvalue of a neutral molecule be equal to the LUMO eigenvalue of the respective cation, and that the HOMO eigenvalue of an anion molecule be equal to the LUMO eigenvalue of the neutral one, respectively. However, determining the screening on frontier (canonical) orbitals is only meant to act as an average measure of the response of the electronic system at hand. In an orbital-resolved framework, screening should be applied to the individual variational orbitals. Also, in the solid state limit, there is no difference, e.g., in the valence band maximum (VBM) for the neutral one and the conduction band minimum (CBM) for the singly ionized one.

The finite-difference procedure we adopt here for the calculation of the screening coefficient $\alpha_{i}$, corresponding to a given orbital $\varphi_{i}$, takes place in two steps and requires two total-energy calculations corresponding to two different occupations $f_{i}$ of the orbital $\varphi_{i}$. A linear-response approach would be more elegant, and its implementation is under way [37], but the protocol below can always be used when linear-response techniques are not available. The goal is to determine each screening coefficient $\alpha_{i}$ such that the expectation value of the Koopmans Hamiltonian on the variational orbital under consideration is independent on its own occupation:

$$
\begin{aligned}
\left.\frac{d E^{\mathrm{KC}}}{d f_{i}}\right|_{f_{i}=s} & =\left.\left\langle\varphi_{i}\left|\hat{H}^{\mathrm{DFT}}+\alpha_{i} \hat{v}_{i}^{\mathrm{KC}}\right| \varphi_{i}\right\rangle\right|_{f_{i}=s} \\
& =\lambda_{i i}^{\alpha_{i}}=\text { constant in } s,
\end{aligned}
$$

where the first identity is the generalization of the Janak theorem [15] to orbital-density-dependent functionals. In practice, this is achieved through the procedure illustrated below.

Step 1 . For a given value of $f_{i}=s \in[0,1]$, we minimize the $\mathrm{KC}$ total energy starting with a best-guess trial value of $\alpha=\alpha^{(0)}$ identical for all orbitals. In order to avoid $\varphi_{i}$ morphing into the VBM (this would always be the most favorable solution because of the Aufbau principle), $\varphi_{i}$ is kept frozen during the minimization while imposing the standard orthogonality condition with all other orbitals belonging to the same spin channel. For orbitals in the opposite spin channel, a standard optimization of the orbitals is performed. At the end of the optimization, the minimum total energy $E_{i}^{\mathrm{KC}}(s)$ compatible with the constraints imposed is obtained, and the expectation value $\lambda_{i i}^{\alpha^{(0)}}(s)$ of the $\mathrm{KC}$ Hamiltonian on $\varphi_{i}$ is calculated. Typically, we repeat this constrained minimization for two values $s=0$ and $s=1$, with the wave functions initialized to those of the chargeneutral system computed with the trial screening factor $\alpha^{(0)}$.

Step 2. We search for the optimal value of $\alpha_{i}$ for which $\lambda_{i i}^{\alpha_{i}}(0)=\lambda_{i i}^{\alpha_{i}}(1)$. Within a second-order approximation of the total energy as a function of $f_{i}$, and assuming a linear dependence of $\lambda_{i i}$ on $\alpha_{i}$, this condition leads to the following expression for $\alpha_{i}$ :

$$
\alpha_{i}=\alpha^{(0)} \frac{\Delta E_{i}-\left.\left\langle\varphi_{i}\left|H_{i}^{\mathrm{DFT}}\right| \varphi_{i}\right\rangle\right|_{0}}{\lambda_{i i}^{\alpha^{(0)}}(0)-\left.\left\langle\varphi_{i}\left|H_{i}^{\mathrm{DFT}}\right| \varphi_{i}\right\rangle\right|_{0}},
$$

where $\Delta E_{i}=E_{i}^{\mathrm{KC}}(s=1)-E_{i}^{\mathrm{KC}}(s=0)$. This two-step procedure is applied to compute $\alpha_{i}$ for each variational orbital [62]. In practice, the number of calculations for $\alpha_{i}$ can be greatly reduced by exploiting the symmetry of the variational orbitals, e.g., in bulk silicon there is only one kind of variational orbital, similar to a bonding Wannier function. Moreover, because each $\alpha_{i}$ can be computed independently, these calculations can be run trivially in parallel.

It is relevant to note that, because of the finite size of the supercells considered, the variation of $s$ in Step 1 produces a spurious interaction between the additional charge density and its periodic replicas, spoiling both $E_{i}^{\mathrm{KC}}$ and $\lambda_{i i}^{\alpha_{i}}$. In order to overcome this problem, we used threedimensional (3D) real-space countercharge corrections $[63,64]$ for the study of isolated molecules, an imagecharge interaction correction model based on the generalized Makov-Payne method [65] in the case of 3D crystals, and extrapolations in one dimension and countercharges in the other two dimensions for the infinite polyethylene chain (detailed expressions for these corrections are provided in the Supplemental Material [66]).

\section{Localization in Koopmans-compliant functionals, and the thermodynamic limit}

In order to investigate how $\mathrm{KC}$ functionals work on extended systems, we start by calculating the IPs, as the opposite of the HOMO energies, of linear alkane molecules $\left(\mathrm{C}_{n} \mathrm{H}_{2 n+1}\right.$-where $\left.n>1\right)$ of increasing length. In particular, we study how the IPs change as a function of molecular length towards the thermodynamic limit represented by the infinite polyethylene (PE) chain. This example also clarifies the importance of localization when working with $\mathrm{KC}$ functionals.

These alkane chains, with staggered conformation, are studied in orthorhombic supercells with at least $15 \AA$ of separation in each direction; to study the infinite polyethylene chain, we consider a supercell containing a $\mathrm{C}_{n} \mathrm{H}_{2 n}$ $(n=22)$ repeat unit $(\simeq 28.5 \AA$ long), while using a $\Gamma$-point sampling of the Brillouin zone. This is equivalent to a $1 \times$ $1 \times 11 \mathbf{k}$-point mesh sampling of the Brilloiun zone for the 


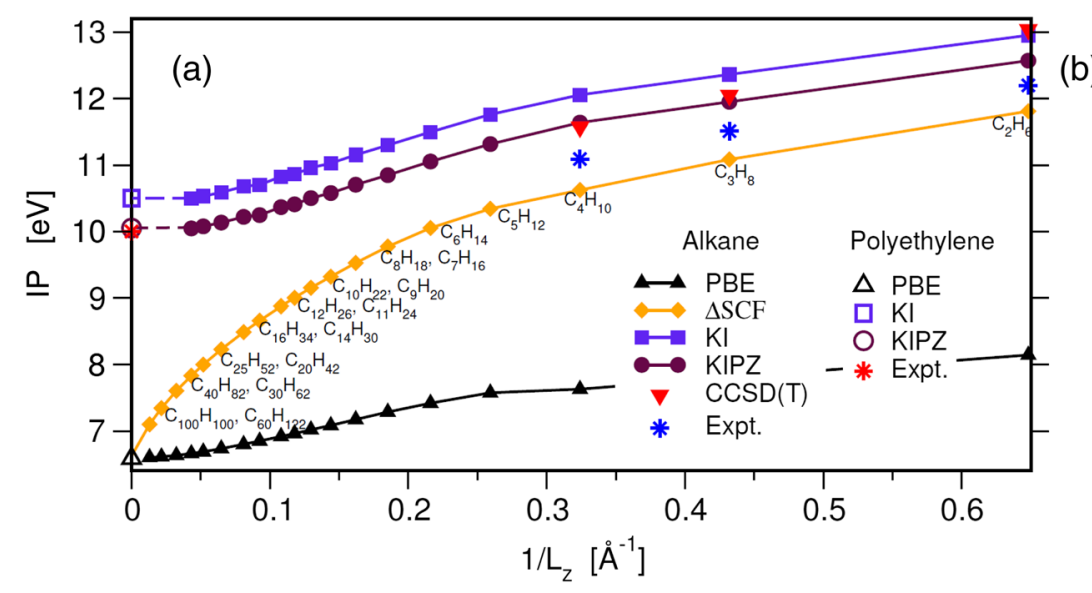

(b)

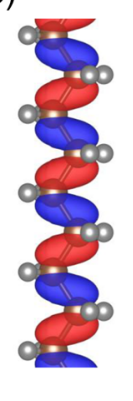

(c)

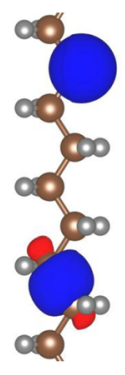

FIG. 1. (a): IP energy as negative of the HOMO energy for linear alkane molecules as a function of the inverse of the system length ( $L_{z}$ in $\AA$ ) and polyethylene (infinite chain), computed with DFT-PBE, $\Delta$ SCF, KI, and KIPZ. $\Delta$ SCF and CCSD(T) results refer to IPs calculated as total energy differences evaluated at the PBE and $\operatorname{CCSD}(\mathrm{T})$ level, respectively; the $\Delta \mathrm{SCF}$ results for $L_{z} \rightarrow \infty$ are an extrapolation. The right panels show (b) the VBM (canonical) orbital of polyethylene computed with DFT-PBE and (c) two types of variational orbitals for the same system computed using KIPZ. For these model systems, geometries were not optimized.

primitive cell of formula unit $\mathrm{C}_{2} \mathrm{H}_{4}$. Details on the convergence of the IP as a function of vacuum size along these directions, as well as on the correction for the finite-cell effects in the $z$-direction when computing the screening factors $\alpha_{i}$, are discussed in Supplemental Material [66]. Calculations have been performed in a plane-wave basis set using norm-conserving pseudopotentials [67] to describe ion-electron interactions. The kinetic energy cutoff for wave functions has been set to $80 \mathrm{Ry}$.

We show in Fig. 1 the IP energies for $19 \mathrm{C}_{n} \mathrm{H}_{2 n+1}$ alkanes ( $n$ going from 2 to 100) and for the extended polyethylene chain, computed using DFT-PBE, KI, and KIPZ (the IP energy is taken as the negative of the KS-HOMO energy or the KS-VBM with respect to the vacuum level). For all molecules, we also provide the $\triangle \mathrm{SCF}$ results, where the IP is computed as the total energy difference between the neutral molecule and its cation, both at the PBE level. Experimental and $\operatorname{CCSD}(\mathrm{T})$ results are also shown (these latter are only available for the three smallest alkanes) [68]. We find that the $\mathrm{KI}$ and KIPZ mean absolute errors (MAE) with respect to experiments are about 0.86 and $0.45 \mathrm{eV}$, respectively. This accuracy is comparable with that of $\operatorname{CCSD}(\mathrm{T})$, which has a MAE of about $0.62 \mathrm{eV}$. In contrast, DFT-PBE significantly underestimates the IP for these molecules with a MAE of $3.74 \mathrm{eV}$, reflecting the intrinsic self-interaction error present in this functional.

As expected, the performance of $\triangle \mathrm{SCF}$ shows a strong dependence on system size. For the three smallest molecules, $\Delta \mathrm{SCF}$ predicts IPs with an accuracy (MAE $=0.43 \mathrm{eV}$ ) equivalent to that of $\mathrm{KC}$ functionals. However, increasing the size of the alkanes, the discrepancies become more and more significant, and, as is well known, when approaching the thermodynamic limit, the $\triangle \mathrm{SCF}$ IP reduces to the PBE one [12-14,69]. The failure of $\Delta \mathrm{SCF}$ in the thermodynamic limit has been discussed extensively, e.g., for silicon nanocrystals (see Ref. [69] and associated discussion) and for hydrogen chains [20]; some of the subtler reasons related to its application to the exact or approximate (e.g., local) functionals are still debated [20,70,71]. In a nutshell, in an approximate functional and as the length of the molecule increases, the HOMO orbital becomes delocalized along the chain [see Fig. 1(b)]. Removing an electron from this orbital-which is exactly what happens in a $\triangle \mathrm{SCF}$ calculation - only slightly modifies the local value of the charge density, since the orbital is normalized to 1 when integrating over the entire system. In the limit of an infinite system, the $\Delta$ SCF IP then reduces to the derivative of the total energy with respect to the particle number [20], which, for a local or semilocal density-functional approximation, is the negative of the KS-DFT HOMO eigenvalue [72] (note that, for the exact functional, the IP would be correct, as would be the band gap calculated as $\left.E_{N+1}+E_{N-1}-2 E_{N}[13,69]\right)$.

Two possible routes to overcome these limitations are, e.g., going beyond the local or semilocal nature of the approximate functionals (e.g., having a nonlocal second derivative with respect to the density $[73,74]$ or modeling the discontinuity of the KS potential $[75,76]$ ) or retaining the simplicity of standard density-functional approximations and working in a localized representation of the orbitals. The dielectric screening localization suggested by Chan and Ceder [77], leading indeed to satisfactory prediction of fundamental band gaps in solids, is an example of the feasibility of this second route. Since, in KC functionals, the generalized Koopmans condition is imposed on the variational orbitals, which are localized [as shown in Fig. 1(c)], rather than the canonical ones, a nonzero correction is present also in the thermodynamic limit; this is the central message of the paper. In fact, KI and KIPZ calculations predict the IP energy of polyethylene to be 10.50 and $10.07 \mathrm{eV}$, respectively, in very good agreement with early 
experimental estimates [78] (about $10.0 \mathrm{eV}$ ); PBE underestimates these values by about $3.5 \mathrm{eV}$.

\section{RESULTS AND DISCUSSION}

\section{A. Band energies for solids}

Having described how KC functionals work in extended systems, we apply such formalism to predict the band gap $E_{g}$ for a set of 30 compounds, including small gap semiconductors and large gap insulators for which accurate experimental and theoretical reference results are available. In Fig. 2 and Table I, we show the accuracy of the KC methods as compared with experiments, standard DFT$\mathrm{PBE}$, and state-of-the-art many-body perturbation theory methods. The zero-point contribution is removed from the experimental data, when available (see the Supplemental Material [66] for the complete list). The results show that in $\mathrm{PBE}$ the value of $E_{g}$ is underestimated with a MAE and mean absolute percent error (MAPE) with respect to experiments of about $2.5 \mathrm{eV}$ and $50 \%$, respectively. Notably, MAEs in KI and KIPZ are down to 0.27 and $0.22 \mathrm{eV}$; this latter is comparable with that obtained by quasiparticle self-consistent $\mathrm{GW}$ with vertex corrections in the polarizability [these are accounted for by an effective exchange-correlation kernel $f_{x c}$ obtained from the BetheSalpeter equation (BSE) [79], or from the bootstrap approximation [80] ], and more than twice as accurate as $\mathrm{G}_{0} \mathrm{~W}_{0}[\mathrm{PBE}][80,81]$, which has a MAE of $0.56 \mathrm{eV}$. In the present calculations, the experimental lattice constants are used for consistency with the literature; also, a supercell technique is used where the Brillouin zone integration is sampled only at the $\Gamma$ point. The band gap is estimated as the energy difference between the highest occupied and the

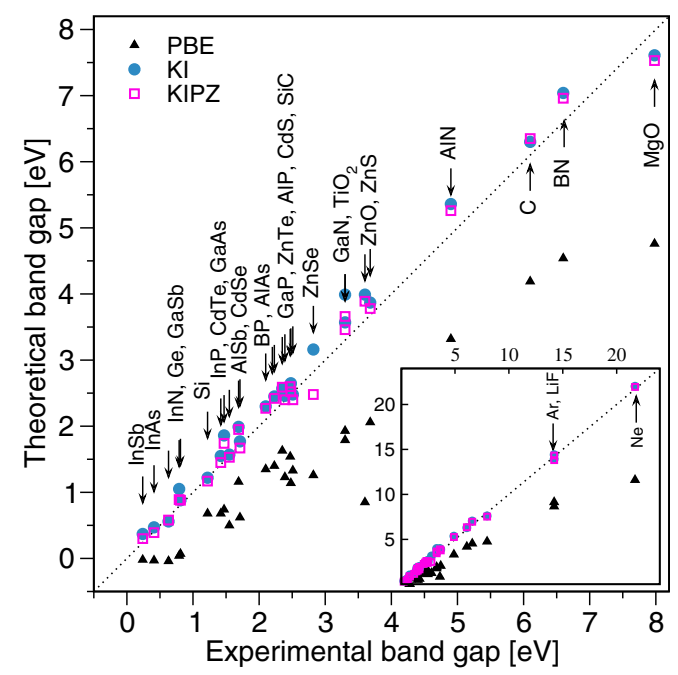

FIG. 2. Band gaps of 30 semiconductors and insulators, calculated using PBE, KI, or KIPZ functionals, compared with available experimental data, shown in an energy range between -0.5 and $8.2 \mathrm{eV}$. A wider energy range (up to $22 \mathrm{eV}$ ) is shown in the inset. lowest unoccupied state at this point. The band gap convergence with respect to supercell size has been tested, yielding an error bar smaller than $0.06 \mathrm{eV}$. Detailed convergence studies with respect to supercell size and cutoff energy for the plane wave expansion of wave functions for each system are presented in the Supplemental Material [66].

To further stress how $\mathrm{KC}$ functionals perform in correcting the band gap of extended systems, we consider $\mathrm{ZnO}$ as a paradigmatic case study: this system is known to be a difficult case to deal with at the GW level, requiring a large number of empty states and dense $\mathbf{k}$-point sampling for the calculation of quasiparticle corrections $[85,86]$. For this system, the use of KC functionals has two main effects: first, $d$ states are shifted to a more accurate position with respect to the top of the valence band, and second, the fundamental gap is enlarged with respect to that of PBE, in much better agreement with experiments. The KI and KIPZ predictions of $E_{g}$ for $\mathrm{ZnO}$ are about 3.96 and $3.76 \mathrm{eV}$, respectively, close to the experimental value of $3.6 \mathrm{eV}$ when the zero-point renormalization correction is considered (3.44 eV otherwise). At the end of the $\mathrm{KC}$ minimization, three classes of variational orbitals are found, corresponding also to three different $\alpha_{i}$ screening values: the $d$-like orbitals centered on $\mathrm{Zn}$ atoms, the $\sigma$-like orbitals localized near $\mathrm{O}$ atoms, and the $\sigma^{*}$-like orbitals near $\mathrm{Zn}$. From the analysis of the projected DOS, we find that, with the optimal $\alpha_{i}$ values, the energy levels of the $d$-band center computed by KI and KIPZ are about $6.81 \mathrm{eV}$ and $7.00 \mathrm{eV}$ with respect to the VBM, close to the experimental values $(7.50-8.81 \mathrm{eV})$; for reference, the PBE prediction is $5.10 \mathrm{eV}$ and the DFT $+U$ is $9.00 \mathrm{eV}$ [87]. Figure 3 shows the density of states (DOS) of $\mathrm{ZnO}$ as predicted by $\mathrm{KI}$ and KIPZ and highlights the good agreement with the experimental ultraviolet photoemission spectrum [82].

Overall, we find that KIPZ performs slightly better than $\mathrm{KI}$ in predicting $E_{g}$. This can be explained by the fact that the KIPZ functional is able to modify not only the electronic excitation energies of approximate DFT functionals, but also the manifold of electronic orbitals (i.e., the

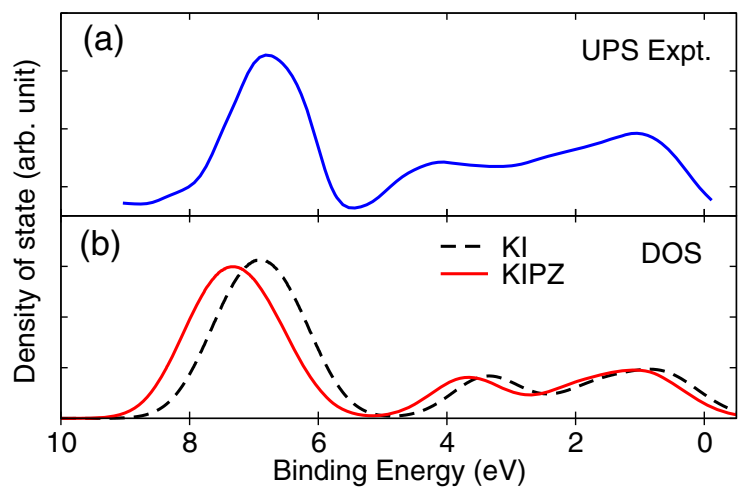

FIG. 3. Density of states (DOS) of $\mathrm{ZnO}$ computed with (b) the $\mathrm{KI}$ and KIPZ functionals, in comparison with (a) the experimental ultraviolet photoemission spectra (UPS expt.) [82]. 
single-particle KS density-matrix) [30]. In particular, a more accurate spatial decay of the density matrix is usually expected as a result of imposing PWL via KIPZ [30]. It is relevant to note that a side effect of having a finite variational PZ term in KIPZ (or infinitesimal in $\mathrm{KI}$ ) is a small symmetry breaking and splitting of the $d$ levels; this unphysical broken symmetry of the $d$-orbitals is driven by the PZ functional and its lack of rotational invariance [54] (in this case, it does not even affect the band gap of the system since it only comes from the $s$ - and $p$-like orbitals [88]).

\section{B. Surfaces: Determination of band edges}

Besides the fundamental gap, the accurate determination of band edge positions is also very important, affecting, e.g., the band alignment at interfaces. In practice, band edge positions cannot be extracted directly from periodic bulk calculations, since an absolute energy reference is needed. A viable solution is to make reference to the ionization potential calculated through the use of surface slabs. This IP is formally defined as the energy difference between the vacuum level $E_{\mathrm{vac}}$ and the VBM. To simplify the convergence of results with respect to the slab thickness, the VBM is determined in a separate bulk calculation and referenced to a local reference potential $V_{\text {ref }}^{b}$, which corresponds to the sum of the electrostatic potential and the local pseudopotential term $[89,90]$. The IP is, therefore, calculated as

$$
\mathrm{IP}=\left(E_{\mathrm{vac}}-V_{\mathrm{ref}}^{s}\right)-\left(\epsilon_{\mathrm{VBM}}^{b}-V_{\mathrm{ref}}^{b}\right),
$$

where the superscript " $s$ " (" $b$ ") refers to slab (bulk) calculations. The slab system is taken sufficiently thick so that the local reference potential inside the slab $V_{\text {ref }}^{s}$ corresponds to $V_{\text {ref }}^{b}$. In practice, $E_{\text {vac }}$ and $V_{\text {ref }}^{s}$ are commonly determined at the PBE level, which has been shown to be reasonably accurate in comparison with higher-level methods such as hybrid functionals [91,92] or GW [93].

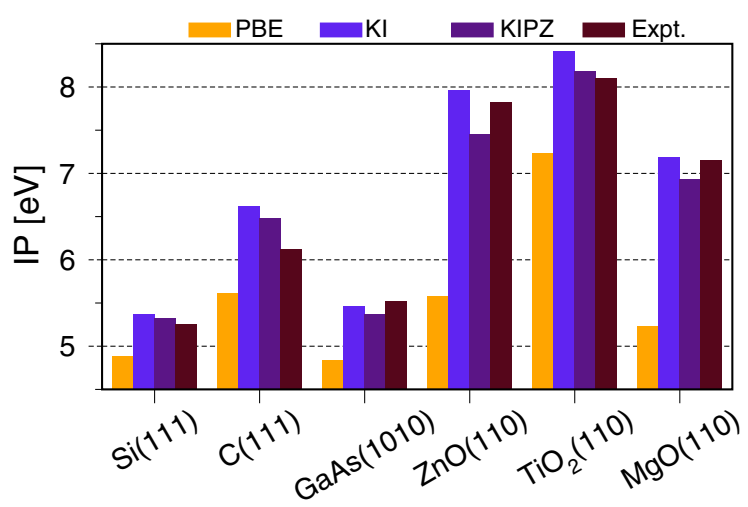

FIG. 4. Ionization potential (in eV) of six different surfaces, including $\mathrm{Si}(111), \mathrm{C}(111), \mathrm{GaAs}(1010), \mathrm{ZnO}(10 \overline{1} 0), \mathrm{TiO}_{2}(110)$, and $\mathrm{MgO}(110)$, calculated using PBE, KI, and KIPZ. The results are compared to experimental values taken from Ref. [80].
TABLE I. Mean absolute errors (MAE, in eV) and mean absolute percent errors (MAPEs, in percent) with respect to experiments for (i) band gaps of 16 solids presented in Fig. 2 for which experimental, $\mathrm{G}_{0} \mathrm{~W}_{0}$, and quasiparticle self-consistent $\mathrm{GW}$ with approximate vertex-correction (QSGW) data are available (see Table II of the Supplemental Material [66] and Refs. [79-81]), and (ii) IP of six surfaces presented in Fig. 4. Experimental values for solid band gap and surfaces are taken from Refs. [80,82-84], respectively.

\begin{tabular}{llrrccc}
\hline \hline & & PBE & $\mathrm{G}_{0} \mathrm{~W}_{0}$ & $\mathrm{KI}$ & $\mathrm{KIPZ}$ & $\mathrm{QSGW}$ \\
\hline$E_{\mathrm{g}}$ & MAE (eV) & 2.54 & 0.56 & 0.27 & 0.22 & 0.18 \\
& MAPE (\%) & 48.28 & 12.10 & 7.09 & 5.37 & 4.46 \\
$\mathrm{IP}$ & MAE (eV) & 1.09 & 0.39 & 0.19 & 0.21 & 0.49 \\
& MAPE (\%) & 15.58 & 5.71 & 2.99 & 3.14 & 7.41 \\
\hline \hline
\end{tabular}

Using the above definitions, we carried out calculations of IPs for six different surfaces, including Si(111), C(111), $\mathrm{GaAs}(1010), \mathrm{ZnO}(10 \overline{1} 0), \mathrm{TiO}_{2}(110)$, and $\mathrm{MgO}(110)$. For comparison, we have used the same surface geometries as in Ref. [80]: 13 atomic layers for $\operatorname{GaAs}(1010), 12$ atomic layers for the $\mathrm{TiO}_{2}$ and $\mathrm{MgO}$ (110) surfaces, and 24 atomic layers for the (111) reconstructed surface of $\mathrm{Si}, \mathrm{Ge}$, and diamond. The thickness of the vacuum region $(25 \AA)$ has been chosen to ensure that there are no interactions between periodic images, and it has been kept the same for all slabs. Results are reported in Fig. 4 and Table I, showing that the accuracy of the $\mathrm{KC}$ functionals is not only much higher than PBE, but also compares favorably with the $\mathrm{G}_{0} \mathrm{~W}_{0}$ and QSGW methods (performed on the same geometries [80]), being basically twice as accurate. As mentioned, QSGẼ uses an approximate bootstrap exchange-correlation kernel [80] to account for vertex corrections in the polarizability. We note that $\mathrm{KC}$ functionals perform equally well for both IPs and band gaps, with a MAE of around $0.2 \mathrm{eV}$ for the KIPZ functional (see Table I). Within the diagrammatic technique, the inclusion of the vertex correction in the polarizability alone is adequate for band gaps, but not enough for IPs, for which further improvements could be achieved by the inclusion of vertex corrections also in the self-energy [94]. It is noteworthy that the KI method does not change the ground-state density of the base functional, making KI $E_{\mathrm{vac}}$ and $V_{\text {ref }}^{s}$ identical to those computed at the PBE level. This is not true for the KIPZ functional and, to overcome this inconsistency, one might compute also $E_{\text {vac }}$ and $V_{\text {ref }}^{s}$ at the KIPZ level; work in this direction could be considered for a future study.

\section{CONCLUSIONS}

We have investigated Koopmans-compliant functionals in the case of extended systems, comparing the results with experimental data and state-of-the-art many-body perturbation theory for a broad range of well-characterized semiconductors and insulators. In doing so, we have developed an approach to compute orbital-dependent screening 
coefficients for $\mathrm{KC}$ functionals with finite differences, and we have applied it to predict IPs and band gaps for both finite and extended systems. First, we have discussed KC functionals for the case of one-dimensional systems with increasing size, as the thermodynamic limit is reached. This analysis has reiterated the importance of imposing the criterion of piecewise linearity on localized orbitals, at variance with the $\triangle \mathrm{SCF}$ approach, which can only use canonical orbitals to predict IPs and EAs and breaks down in extended systems. Then, we have studied reference solids and shown that the KI and KIPZ functionals can yield very accurate results for band gaps of different semiconductors and insulators, with mean absolute errors that are of the order of $0.2 \mathrm{eV}$, and comparable with the most accurate QSGW. The comparison is even more favorable for the IPs studied, with an accuracy doubled with respect to QSGW. These results are more remarkable considering that the fundamental ingredient in our functionals remains the PBE generalizedgradient approximation, and they have been obtained with a functional theory of the occupied states. Their accuracy and simplicity, given the computational costs broadly comparable to standard density-functional theory, make $\mathrm{KC}$ functionals very attractive for studying electronic levels in complex materials and devices. It also reiterates the suggestion that charged excitations, such as electron additions and removals, can be studied not only with diagrammatic approaches, such as GW, but with functional theories that are dynamical (i.e., frequency dependent) and even local (as discussed in Refs. [33,39]), with $\mathrm{KC}$ functionals taking the role of approximated spectral functionals, i.e., able to reproduce not only the total energy, but also the spectral properties of the system at hand.

\section{ACKNOWLEDGMENTS}

We acknowledge partial support from the Swiss National Centre for Computational Design and Discovery of Novel Materials (MARVEL) and from the EU Centre of Excellence "MaX-Materials Design at the Exascale" (Horizon 2020 EINFRA-5, Grant No. 676598). We would like to thank Prof. Ismaila Dabo, Dr. Marco Gibertini, and Dr. Fabien Bruneval for useful discussions, and Dr. Wei Chen and Professor Alfredo Pasquarello for providing the surface geometries used in Sec. III B.

[1] T. A. Pham, Y. Ping, and G. Galli, Modelling Heterogeneous Interfaces for Solar Water Splitting, Nat. Mater. 16, 401 (2017).

[2] G. Onida, L. Reining, and A. Rubio, Electronic Excitations: Density-Functional Versus Many-Body Green's-Function Approaches, Rev. Mod. Phys. 74, 601 (2002).
[3] L. Hedin, New Method for Calculating the One-Particle Green's Function with Application to the Electron-Gas Problem, Phys. Rev. 139, A796 (1965).

[4] J. McClain, Q. Sun, G. K.-L. Chan, and T. C. Berkelbach, Gaussian-Based Coupled-Cluster Theory for the GroundState and Band Structure of Solids, J. Chem. Theory Comput. 13, 1209 (2017).

[5] W. M. C. Foulkes, L. Mitas, R. J. Needs, and G. Rajagopal, Quantum Monte Carlo Simulations of Solids, Rev. Mod. Phys. 73, 33 (2001).

[6] P. Hohenberg and W. Kohn, Inhomogeneous Electron Gas, Phys. Rev. 136, B864 (1964).

[7] W. Kohn and L. J. Sham, Self-Consistent Equations Including Exchange and Correlation Effects, Phys. Rev. 140, A1133 (1965).

[8] A. D. Becke, A New Mixing of Hartree-Fock and Local Density-Functional Theories, J. Chem. Phys. 98, 1372 (1993).

[9] M. Levy, J. P. Perdew, and V. Sahni, Exact Differential Equation for the Density and Ionization Energy of a ManyParticle System, Phys. Rev. A 30, 2745 (1984).

[10] C.-O. Almbladh and U. von Barth, Exact Results for the Charge and Spin Densities, Exchange-Correlation Potentials, and Density-Functional Eigenvalues, Phys. Rev. B 31, 3231 (1985).

[11] J. P. Perdew and M. Levy, Comment on Significance of the Highest Occupied Kohn-Sham Eigenvalue, Phys. Rev. B 56, 16021 (1997).

[12] J. P. Perdew and M. Levy, Physical Content of the Exact Kohn-Sham Orbital Energies: Band Gaps and Derivative Discontinuities, Phys. Rev. Lett. 51, 1884 (1983).

[13] L. J. Sham and M. Schlüter, Density-Functional Theory of the Energy Gap, Phys. Rev. Lett. 51, 1888 (1983).

[14] J. P. Perdew, W. Yang, K. Burke, Z. Yang, E. K. U. Gross, M. Scheffler, G. E. Scuseria, T. M. Henderson, I. Y. Zhang, A. Ruzsinszky, H. Peng, J. Sun, E. Trushin, and A. Görling, Understanding Band Gaps of Solids in Generalized Kohn-Sham Theory, Proc. Natl. Acad. Sci. U.S.A. 114, 2801 (2017).

[15] J.F. Janak, Proof that $\left[(\partial E) /\left(\partial n_{i}\right)\right]=\epsilon$ in DensityFunctional Theory, Phys. Rev. B 18, 7165 (1978).

[16] M. Cococcioni and S. de Gironcoli, Linear Response Approach to the Calculation of the Effective Interaction Parameters in the LDA + U Method, Phys. Rev. B 71, 035105 (2005).

[17] H. J. Kulik, M. Cococcioni, D. A. Scherlis, and N. Marzari, Density Functional Theory in Transition-Metal Chemistry: A Self-Consistent Hubbard U Approach, Phys. Rev. Lett. 97, 103001 (2006).

[18] P. Mori-Sánchez, A. J. Cohen, and W. Yang, Many-Electron Self-Interaction Error in Approximate Density Functionals, J. Chem. Phys. 125, 201102 (2006).

[19] A. J. Cohen, P. Mori-Sánchez, and W. Yang, Insights into Current Limitations of Density Functional Theory, Science 321, 792 (2008).

[20] P. Mori-Sánchez, A. J. Cohen, and W. Yang, Localization and Delocalization Errors in Density Functional Theory and Implications for Band-Gap Prediction, Phys. Rev. Lett. 100, 146401 (2008). 
[21] J. P. Perdew and A. Zunger, Self-Interaction Correction to Density-Functional Approximations for Many-Electron Systems, Phys. Rev. B 23, 5048 (1981).

[22] T Stein, H. Eisenberg, L. Kronik, and R. Baer, Fundamental Gaps in Finite Systems from Eigenvalues of a Generalized Kohn-Sham Method, Phys. Rev. Lett. 105, 266802 (2010).

[23] S. Refaely-Abramson, S. Sharifzadeh, N. Govind, J. Autschbach, J. B. Neaton, R. Baer, and L. Kronik, Quasiparticle Spectra from a Nonempirical Optimally Tuned Range-Separated Hybrid Density Functional, Phys. Rev. Lett. 109, 226405 (2012).

[24] J. H. Skone, M. Govoni, and G. Galli, Self-Consistent Hybrid Functional for Condensed Systems, Phys. Rev. B 89, 195112 (2014).

[25] N. P. Brawand, M. Vörös, M. Govoni, and G. Galli, Generalization of Dielectric-Dependent Hybrid Functionals to Finite Systems, Phys. Rev. X 6, 041002 (2016).

[26] I. Dabo, M. Cococcioni, and N. Marzari, Non-Koopmans Corrections in Density-Functional Theory: Self-Interaction Revisited, arXiv:0901.2637v1.

[27] I. Dabo, A. Ferretti, N. Poilvert, Y. Li, N. Marzari, and M. Cococcioni, Koopmans Condition for Density-Functional Theory, Phys. Rev. B 82, 115121 (2010).

[28] I. Dabo, A. Ferretti, G. Borghi, N. L. Nguyen, N. Poilvert, C. H. Park, M. Cococcioni, and N. Marzari, Piecewise Linearity and Spectroscopic Properties from KoopmansCompliant Functionals, Psi-K Newsletter 119, 1 (2013).

[29] I. Dabo, A. Ferretti, C. H. Park, N. Poilvert, Y. Li, M. Cococcioni, and N. Marzari, Donor and Acceptor Levels of Organic Photovoltaic Compounds from First Principles, Phys. Chem. Chem. Phys. 15, 685 (2013).

[30] G. Borghi, A. Ferretti, N. L. Nguyen, I. Dabo, and N. Marzari, Koopmans-Compliant Functionals and Their Performance against Reference Molecular Data, Phys. Rev. B 90, 075135 (2014).

[31] N. L. Nguyen, G. Borghi, A. Ferretti, I. Dabo, and N. Marzari, First-Principles Photoemission Spectroscopy and Orbital Tomography in Molecules from KoopmansCompliant Functionals, Phys. Rev. Lett. 114, 166405 (2015).

[32] N. L. Nguyen, G. Borghi, A. Ferretti, and N. Marzari, FirstPrinciples Photoemission Spectroscopy of DNA and RNA Nucleobases from Koopmans-Compliant Functionals, J. Chem. Theory Comput. 12, 3948 (2016).

[33] A. Ferretti, I. Dabo, M. Cococcioni, and N. Marzari, Bridging Density-Functional and Many-Body Perturbation Theory: Orbital-Density Dependence in ElectronicStructure Functionals, Phys. Rev. B 89, 195134 (2014).

[34] J. P. Perdew, K. Burke, and M. Ernzerhof, Generalized Gradient Approximation Made Simple, Phys. Rev. Lett. 77, 3865 (1996).

[35] G. Borghi, C. H. Park, N. L. Nguyen, A. Ferretti, and N. Marzari, Variational Minimization of Orbital-DensityDependent Functionals, Phys. Rev. B 91, 155112 (2015).

[36] J. P. Perdew, R. G. Parr, M. Levy, and J. L. Balduz, DensityFunctional Theory for Fractional Particle Number: Derivative Discontinuities of the Energy, Phys. Rev. Lett. 49, 1691 (1982).
[37] N. Colonna, N. L. Nguyen, A. Ferretti, and N. Marzari, Screening in Orbital-Density-Dependent Functionals, J. Chem. Theory Comput. 14, 2549 (2018).

[38] N. Marzari, A. A. Mostofi, J. R. Yates, I. Souza, and D. Vanderbilt, Maximally Localized Wannier Functions: Theory and Applications, Rev. Mod. Phys. 84, 1419 (2012).

[39] M. Gatti, V. Olevano, L. Reining, and I. V. Tokatly, Transforming Nonlocality into a Frequency Dependence: A Shortcut to Spectroscopy, Phys. Rev. Lett. 99, 057401 (2007).

[40] I. Dabo, Towards First-Principles Electrochemistry, Ph.D. thesis, MIT, Cambridge, MA (2008), http://dspace.mit.edu/ handle/1721.1/44320.

[41] I. Dabo, A. Ferretti, and N. Marzari, Piecewise Linearity and Spectroscopic Properties from Koopmans-Compliant Functionals, Top. Curr. Chem. 347, 193 (2014).

[42] Note that Eq. (6) is exactly equivalent to Eq. (27) in Ref. [30].

[43] O. A. Vydrov and G. E. Scuseria, Effect of the PerdewZunger Self-Interaction Correction on the Thermochemical Performance of Approximate Density Functionals, J. Chem. Phys. 121, 8187 (2004).

[44] O. A. Vydrov and G. E. Scuseria, Ionization Potentials and Electron Affinities in the Perdew-Zunger Self-Interaction Corrected Density-Functional Theory, J. Chem. Phys. 122, 184107 (2005).

[45] S. Klüpfel, P. Klüpfel, and H. Jónsson, The Effect of the Perdew-Zunger Self-Interaction Correction to Density Functionals on the Energetics of Small Molecules, J. Chem. Phys. 137, 124102 (2012).

[46] O. A. Vydrov, G. E. Scuseria, J. P. Perdew, A. Ruzsinszky, and G. I. Csonka, Scaling Down the Perdew-Zunger SelfInteraction Correction in Many-Electron Regions, J. Chem. Phys. 124, 094108 (2006).

[47] H. Jónsson, Simulation of Surface Processes, Proc. Natl. Acad. Sci. U.S.A. 108, 944 (2011).

[48] E. J. Bylaska, K. Tsemekhman, and F. Gao, New Development of Self-Interaction Corrected DFT for Extended Systems Applied to the Calculation of Native Defects in 3C-SiC, Phys. Scr. 2006, 86 (2006).

[49] E. Bylaska, K. Tsemekhman, and H. Jonsson, SelfConsistent Self-Interaction Corrected DFT: The Method and Applications to Extended and Confined Systems, in APS Meeting Abstracts (American Physical Society, 2004), p. L38.004, http://flux.aps.org/meetings/YR04/MAR04/ baps/abs/S4380.html\#SL38.004.

[50] We note that further refinements could use different screening parameters to impose PWL and to impose correctly the charge transfer or polarizability.

[51] S. Lehtola and H. Jónsson, Variational, Self-Consistent Implementation of the Perdew-Zunger Self-Interaction Correction with Complex Optimal Orbitals, J. Chem. Theory Comput. 10, 5324 (2014).

[52] O. A. Vydrov, G. E. Scuseria, and J. P. Perdew, Tests of Functionals for Systems with Fractional Electron Number, J. Chem. Phys. 126, 154109 (2007).

[53] M. R. Pederson, R. A. Heaton, and C. C. Lin, Local-Density Hartree-Fock Theory of Electronic States of Molecules with Self-Interaction Correction, J. Chem. Phys. 80, 1972 (1984). 
[54] M. Stengel and N. A. Spaldin, Self-Interaction Correction with Wannier Functions, Phys. Rev. B 77, 155106 (2008).

[55] D. Hofmann, S. Klüpfel, P. Klüpfel, and S. Kümmel, Using Complex Degrees of Freedom in the Kohn-Sham SelfInteraction Correction, Phys. Rev. A 85, 062514 (2012).

[56] N. Marzari, D. Vanderbilt, and M. C. Payne, Ensemble Density-Functional Theory for Ab Initio Molecular Dynamics of Metals and Finite-Temperature Insulators, Phys. Rev. Lett. 79, 1337 (1997).

[57] M. R. Pederson and C. C. Lin, Localized and Canonical Atomic Orbitals in Self-Interaction Corrected Local Density Functional Approximation, J. Chem. Phys. 88, 1807 (1988).

[58] The delocalization of empty states is due to (i) the entanglement of the high-lying nearly free electron band (which is very delocalized) and low-lying conduction bands, and (ii) the residual Hartree contribution to empty states' potentials (see the detailed description of the KC potentials in Ref. [30]).

[59] To obtain the maximally localized Wannier orbitals for the low-lying empty states, we employed the disentanglement maximally localized Wannier function technique proposed in Ref. [60]. All the details about the procedure followed can be found in the Supplemental Material.

[60] I. Souza, N. Marzari, and D. Vanderbilt, Maximally Localized Wannier Functions for Entangled Energy Bands, Phys. Rev. B 65, 035109 (2001).

[61] J. Ma and L.W. Wang, Using Wannier Functions to Improve Solid Band Gap Predictions in Density Functional Theory, Sci. Rep. 6, 24924 (2016).

[62] For empty orbitals, the procedure is essentially the same. The only difference is that, in this case, we have an extra (partially) occupied orbital corresponding to the empty orbital in the original $N$-electron system.

[63] I. Dabo, B. Kozinsky, N. E. Singh-Miller, and N. Marzari, Electrostatics in Periodic Boundary Conditions and RealSpace Corrections, Phys. Rev. B 77, 115139 (2008).

[64] Y. Li and I. Dabo, Electronic Levels and Electrical Response of Periodic Molecular Structures from PlaneWave Orbital-Dependent Calculations, Phys. Rev. B 84, 155127 (2011).

[65] G. Makov and M. C. Payne, Periodic Boundary Conditions in Ab Initio Calculations, Phys. Rev. B 51, 4014 (1995).

[66] See the Supplemental Material http://link.aps.org/ supplemental/10.1103/PhysRevX.8.021051 for the detailed expansion of a generalized Makov-Payne model for the finitesize supercell effect corrections used for the calculation of the orbital screening factors $\alpha_{i}$ in the 3D solids, the setup parameter for the supercell calculations, the absolute values of band gaps and surface IP presented in Table 1 and Figs. 2 and 4 , and a method to correct the finite-size supercell effect for computing the IP of 1D polyethylene.

[67] M. Schlipf and F. Gygi, Optimization Algorithm for the Generation of ONCV Pseudopotentials, Comput. Phys. Commun. 196, 36 (2015).

[68] K. Krause, M. E. Harding, and W. Klopper, CoupledCluster Reference Values for the GW27 and GW100 Test Sets for the Assessment of GW Methods, Mol. Phys. 113, 1952 (2015).
[69] R. W. Godby and I. D. White, Density-Relaxation Part of the Self-Energy, Phys. Rev. Lett. 80, 3161 (1998).

[70] A. Görling, Exchange-Correlation Potentials with Proper Discontinuities for Physically Meaningful Kohn-Sham Eigenvalues and Band Structures, Phys. Rev. B 91, 245120 (2015).

[71] V. Vlček, H. R. Eisenberg, G. Steinle-Neumann, L. Kronik, and R. Baer, Deviations from Piecewise Linearity in the Solid-State Limit with Approximate Density Functionals, J. Chem. Phys. 142, 034107 (2015).

[72] A. J. Cohen, P. Mori-Sánchez, and W. Yang, Fractional Charge Perspective on the Band Gap in Density-Functional Theory, Phys. Rev. B 77, 115123 (2008).

[73] E. Kraisler and L. Kronik, Fundamental Gaps with Approximate Density Functionals: The Derivative Discontinuity Revealed from Ensemble Considerations, J. Chem. Phys. 140, 18A540 (2014).

[74] E. Trushin, M. Betzinger, S. Blügel, and A. Görling, Band Gaps, Ionization Potentials, and Electron Affinities of Periodic Electron Systems via the Adiabatic-Connection Fluctuation-Dissipation Theorem, Phys. Rev. B 94, 075123 (2016).

[75] O. Gritsenko, R. van Leeuwen, E. van Lenthe, and E. J. Baerends, Self-Consistent Approximation to the Kohn-Sham Exchange Potential, Phys. Rev. A 51, 1944 (1995).

[76] M. Kuisma, J. Ojanen, J. Enkovaara, and T. T. Rantala, Kohn-Sham Potential with Discontinuity for Band Gap Materials, Phys. Rev. B 82, 115106 (2010).

[77] M. K. Y. Chan and G. Ceder, Efficient Band Gap Prediction for Solids, Phys. Rev. Lett. 105, 196403 (2010).

[78] R. H. Partridge, Vacuum-Ultraviolet Absorption Spectrum of Polyethylene, J. Chem. Phys. 45, 1685 (1966).

[79] M. Shishkin, M. Marsman, and G. Kresse, Accurate Quasiparticle Spectra from Self-Consistent GW Calculations with Vertex Corrections, Phys. Rev. Lett. 99, 246403 (2007).

[80] W. Chen and A. Pasquarello, Accurate Band Gaps of Extended Systems via Efficient Vertex Corrections in GW, Phys. Rev. B 92, 041115 (2015).

[81] M. Shishkin and G. Kresse, Self-Consistent GW Calculations for Semiconductors and Insulators, Phys. Rev. B 75, 235102 (2007).

[82] O. Madelung, Semiconductors: Data Handbook (SpringerVerlag, Berlin, Heidelberg, 2004).

[83] M. Piacentini, D. W. Lynch, and C. G. Olson, Thermoreflectance of LiF between 12 and $30 \mathrm{eV}$, Phys. Rev. B 13, 5530 (1976).

[84] N. Schwentner, F. J. Himpsel, V. Saile, M. Skibowski, W. Steinmann, and E. E. Koch, Photoemission from Rare-Gas Solids: Electron Energy Distributions from the Valence Bands, Phys. Rev. Lett. 34, 528 (1975).

[85] B.-C. Shih, Y. Xue, P. Zhang, M. L. Cohen, and S. G. Louie, Quasiparticle Band Gap of ZnO: High Accuracy from the Conventional $\mathrm{G}^{0} \mathrm{~W}^{0}$ Approach, Phys. Rev. Lett. 105, 146401 (2010).

[86] M. Stankovski, G. Antonius, D. Waroquiers, A. Miglio, H. Dixit, K. Sankaran, M. Giantomassi, X. Gonze, M. Côté, and G.-M. Rignanese, $\mathrm{G}^{0} \mathrm{~W}^{0}$ Band Gap of $\mathrm{ZnO}$ : Effects of Plasmon-Pole Models, Phys. Rev. B 84, 241201 (2011). 
[87] L. A. Agapito, S. Curtarolo, and M. Buongiorno Nardelli, Reformulation of DFT $+\mathrm{U}$ as a Pseudohybrid Hubbard Density Functional for Accelerated Materials Discovery, Phys. Rev. X 5, 011006 (2015).

[88] M. R. Pederson, Fermi Orbital Derivatives in Self-Interaction Corrected Density Functional Theory: Applications to Closed Shell Atoms, J. Chem. Phys. 142, 064112 (2015).

[89] A. Baldereschi, S. Baroni, and R. Resta, Band Offsets in Lattice-Matched Heterojunctions: A Model and FirstPrinciples Calculations for GaAs/AlAs, Phys. Rev. Lett. 61, 734 (1988).

[90] N. E. Singh-Miller and N. Marzari, Surface Energies, Work Functions, and Surface Relaxations of Low-Index Metallic Surfaces from First Principles, Phys. Rev. B 80, 235407 (2009).
[91] A. Alkauskas, P. Broqvist, F. Devynck, and A. Pasquarello, Band Offsets at Semiconductor-Oxide Interfaces from Hybrid Density-Functional Calculations, Phys. Rev. Lett. 101, 106802 (2008).

[92] K. Steiner, W. Chen, and A Pasquarello, Band Offsets of Lattice-Matched Semiconductor Heterojunctions through Hybrid Functionals and $\mathrm{G}_{0} \mathrm{~W}_{0}$, Phys. Rev. B 89, 205309 (2014).

[93] R. Shaltaf, G.-M. Rignanese, X. Gonze, F. Giustino, and A. Pasquarello, Band Offsets at the $\mathrm{Si} / \mathrm{SiO}_{2}$ Interface from Many-Body Perturbation Theory, Phys. Rev. Lett. 100, 186401 (2008).

[94] A. Grüneis, G. Kresse, Y. Hinuma, and F. Oba, Ionization Potentials of Solids: The Importance of Vertex Corrections, Phys. Rev. Lett. 112, 096401 (2014). 